\title{
Complete constant mean curvature surfaces in homogeneous spaces
}

José M. Espinar* and Harold Rosenberg

\begin{abstract}
In this paper we classify complete surfaces of constant mean curvature whose Gaussian curvature does not change sign in a simply connected homogeneous manifold with a 4dimensional isometry group.
\end{abstract}

Mathematics Subject Classification (2010). 53A10, 53C21.

Keywords. Constant mean curvature, homogeneous spaces.

\section{Introduction}

In 1966, T. Klotz and R. Ossermann showed the following:

Theorem ([KO]). A complete $H$-surface in $\mathbb{R}^{3}$ whose Gaussian curvature $K$ does not change sign is either a sphere, a minimal surface, or a right circular cylinder.

The above result was extended to $\mathbb{S}^{3}$ by D. Hoffman $[\mathrm{H}]$, and to $\mathbb{H}^{3}$ by R. Tribuzy [T] with an extra hypothesis if $K$ is non-positive. The additional hypothesis says that, when $K \leq 0$, one has $H^{2}-K-1>0$.

In recent years, the study of $H$-surfaces in product spaces and, more generally, in a homogeneous three-manifold with a 4-dimensional isometry group is quite active (see [AR], [AR2], [CoR], [ER], [FM], [FM2], [DH] and references therein).

The aim of this paper is to extend the above theorem to homogeneous spaces with a 4-dimensional isometry group. These homogeneous spaces are denoted by $\mathbb{E}(\kappa, \tau)$, where $\kappa$ and $\tau$ are constant and $\kappa-4 \tau^{2} \neq 0$. They can be classified as $\mathbb{M}^{2}(\kappa) \times \mathbb{R}$ if $\tau=0$, with $\mathbb{M}^{2}(\kappa)=\mathbb{S}^{2}(\kappa)$ if $\kappa>0\left(\mathbb{S}^{2}(\kappa)\right.$ the sphere of curvature $\left.\kappa\right)$, and $\mathbb{M}^{2}(\kappa)=\mathbb{H}^{2}(\kappa)$ if $\kappa<0\left(\mathbb{H}^{2}(\kappa)\right.$ the hyperbolic plane of curvature $\left.\kappa\right)$. If $\tau$ is not equal to zero, $\mathbb{E}(\kappa, \tau)$ is a Berger sphere if $\kappa>0$, a Heisenberg space if $\kappa=0$ (of

\footnotetext{
* The author is partially supported by Spanish MEC-FEDER Grant MTM2007-65249, and Regional J. Andalucia Grants P06-FQM-01642 and FQM325.
} 
bundle curvature $\tau$ ), and the universal cover of $\operatorname{PSL}(2, \mathbb{R})$ if $\kappa<0$. Henceforth we will suppose $\kappa$ is plus or minus one or zero.

The paper is organized as follows. In Section 2, we establish the definitions and necessary equations for an $H$-surface. We also state here two classification results for $H$-surfaces. We prove them in Section 5 and Section 6 for the sake of completeness.

Section 3 is devoted to the classification of $H$-surfaces with non-negative Gaussian curvature,

Theorem 3.1. Let $\Sigma \subset \mathbb{E}(\kappa, \tau)$ be a complete $H$-surface with $K \geq 0$. Then, $\Sigma$ is either a rotational sphere (in particular, $4 H^{2}+\kappa>0$ ), or a complete vertical cylinder over a complete curve of geodesic curvature $2 \mathrm{H}$ on $\mathbb{M}^{2}(\kappa)$.

In Section 4 we continue with the classification of $H$-surfaces with non-positive Gaussian curvature.

Theorem 4.1. Let $\Sigma \subset \mathbb{E}(\kappa, \tau)$ be a complete $H$-surface with $K \leq 0$ and $H^{2}+$ $\tau^{2}-\left|\kappa-4 \tau^{2}\right|>0$. Then, $\Sigma$ is a complete vertical cylinder over a complete curve of geodesic curvature $2 H$ on $\mathbb{M}^{2}(\kappa)$.

The above theorem is not true without the inequality; for example, any complete minimal surface in $\mathbb{H}^{2} \times \mathbb{R}$ that is not a vertical cylinder.

In the Appendix, we give a result, which we think is of independent interest, concerning differential operators on a Riemannian surface $\Sigma$ of the form $\Delta+g$, acting on $C^{2}(\Sigma)$-functions, where $\Delta$ is the Laplacian with respect to the Riemannian metric on $\Sigma$ and $g \in C^{0}(\Sigma)$.

\section{The geometry of surfaces in homogeneous spaces}

Henceforth $\mathbb{E}(\kappa, \tau)$ denotes a complete simply connected homogeneous three-manifold with 4-dimensional isometry group. Such a three-manifold can be classified in terms of a pair of real numbers $(\kappa, \tau)$ satisfying $\kappa-4 \tau^{2} \neq 0$. In fact, these manifolds are Riemannian submersions over a complete simply-connected surface $\mathbb{M}^{2}(\kappa)$ of constant curvature $\kappa, \pi: \mathbb{E}(\kappa, \tau) \rightarrow \mathbb{M}^{2}(\kappa)$, and translations along the fibers are isometries, therefore they generate a Killing field $\xi$, called the vertical field. Moreover, $\tau$ is the real number such that $\bar{\nabla}_{X} \xi=\tau X \wedge \xi$ for all vector fields $X$ on the manifold. Here, $\bar{\nabla}$ is the Levi-Civita connection of the manifold and $\wedge$ is the cross product.

Let $\Sigma$ be a complete $H$-surface immersed in $\mathbb{E}(\kappa, \tau)$. By passing to a 2 -sheeted covering space of $\Sigma$, we can assume $\Sigma$ is orientable. Let $N$ be a unit normal to $\Sigma$. In terms of a conformal parameter $z$ of $\Sigma$, the first, $\langle\cdot, \cdot\rangle$, and second, $I I$, fundamental 
forms are given by

$$
\begin{aligned}
& \langle\cdot, \cdot\rangle=\lambda|d z|^{2} \\
& I I=p d z^{2}+\lambda H|d z|^{2}+\bar{p} d \bar{z}^{2},
\end{aligned}
$$

where $p d z^{2}=\left\langle-\nabla_{\partial_{z}} N, \partial_{z}\right\rangle d z^{2}$ is the Hopf differential of $\Sigma$.

Set $v=\langle N, \xi\rangle$ and $T=\xi-v N$, i.e., $v$ is the normal component of the vertical field $\xi$, called the angle function, and $T$ is the tangent component of the vertical field.

First we state the following necessary equations on $\Sigma$ which were obtained in [FM].

Lemma 2.1. Given an immersed surface $\Sigma \subset \mathbb{E}(\kappa, \tau)$, the following equations are satisfied:

$$
\begin{aligned}
K & =K_{e}+\tau^{2}+\left(\kappa-4 \tau^{2}\right) v^{2}, \\
p_{\bar{z}} & =\frac{\lambda}{2}\left(H_{z}+\left(\kappa-4 \tau^{2}\right) v A\right), \\
A_{\bar{z}} & =\frac{\lambda}{2}(H+i \tau) \nu, \\
v_{z} & =-(H-i \tau) A-\frac{2}{\lambda} p \bar{A}, \\
|A|^{2} & =\frac{1}{4} \lambda\left(1-v^{2}\right), \\
A_{z} & =\frac{\lambda_{z}}{\lambda} A+p v,
\end{aligned}
$$

where $A=\left\langle\xi, \partial_{z}\right\rangle, K_{e}$ the extrinsic curvature and $K$ the Gauss curvature of $\Sigma$.

For an immersed $H$-surface $\Sigma \subset \mathbb{E}(\kappa, \tau)$ there is a globally defined quadratic differential, called the Abresch-Rosenberg differential, which in these coordinates is given by (see [AR2]):

$$
Q d z^{2}=\left(2(H+i \tau) p-\left(\kappa-4 \tau^{2}\right) A^{2}\right) d z^{2},
$$

following the notation above.

It is not hard to verify this quadratic differential is holomorphic on an $H$-surface using (2.3) and (2.4),

Theorem 2.1 ([AR], [AR2]). $Q d z^{2}$ is a holomorphic quadratic differential on any $H$-surface in $\mathbb{E}(\kappa, \tau)$.

Associated to the Abresch-Rosenberg differential we define the smooth function $q: \Sigma \rightarrow[0,+\infty)$ given by

$$
q=\frac{4|Q|^{2}}{\lambda^{2}}
$$


By means of Theorem 2.1, $q$ either has isolated zeroes or vanishes identically. Note that $q$ does not depend on the conformal parameter $z$, hence $q$ is globally defined on $\Sigma$.

We continue this section establishing some formulae relating the angle function, $q$ and the Gaussian curvature.

Lemma 2.2. Let $\Sigma$ be an $H$-surface immersed in $\mathbb{E}(\kappa, \tau)$. Then the following equations are satisfied:

$$
\begin{gathered}
\|\nabla v\|^{2}=\frac{4 H^{2}+\kappa-\left(\kappa-4 \tau^{2}\right) v^{2}}{4\left(\kappa-4 \tau^{2}\right)}\left(4\left(H^{2}-K_{e}\right)\right. \\
\left.+\left(\kappa-4 \tau^{2}\right)\left(1-v^{2}\right)\right)-\frac{q}{\kappa-4 \tau^{2}}, \\
\Delta v=-\left(4 H^{2}+2 \tau^{2}+\left(\kappa-4 \tau^{2}\right)\left(1-v^{2}\right)-2 K_{e}\right) v .
\end{gathered}
$$

Moreover, away from the isolated zeroes of $q$, we have

$$
\Delta \ln q=4 K \text {. }
$$

Proof. From (2.5)

$$
\left|v_{z}\right|^{2}=\frac{4|p|^{2}|A|^{2}}{\lambda^{2}}+\left(H^{2}+\tau^{2}\right)|A|^{2}+\frac{2(H+i \tau)}{\lambda} p \bar{A}^{2}+\frac{2(H-i \tau)}{\lambda} \bar{p} A^{2},
$$

and taking into account that

$$
\begin{aligned}
|Q|^{2}=4 & \left(H^{2}+\tau^{2}\right)|p|^{2}+\left(\kappa-4 \tau^{2}\right)^{2}|A|^{4}-\left(\kappa-4 \tau^{2}\right)\left(2(H+i \tau) p \bar{A}^{2}\right. \\
& \left.+2(H-i \tau) \bar{p} A^{2}\right),
\end{aligned}
$$

we obtain, using also (2.6), that

$$
\begin{aligned}
\left|v_{z}\right|^{2}=( & \left.H^{2}+\tau^{2}\right)|A|^{2}+\left(H^{2}-K_{e}\right)|A|^{2}+\left(\kappa-4 \tau^{2}\right) \frac{|A|^{4}}{\lambda} \\
& +4\left(\frac{H^{2}+\tau^{2}}{\kappa-4 \tau^{2}}\right) \frac{|p|^{2}}{\lambda}-\frac{|Q|^{2}}{\left(\kappa-4 \tau^{2}\right) \lambda}
\end{aligned}
$$

where we have used that $4|p|^{2}=\lambda^{2}\left(H^{2}-K_{e}\right)$ and $\kappa-4 \tau^{2} \neq 0$. Thus

$$
\begin{gathered}
\|\nabla v\|^{2}=\frac{4}{\lambda}\left|v_{z}\right|^{2}=\left(2 H^{2}-K_{e}+\tau^{2}\right)\left(1-v^{2}\right)+\frac{\kappa-4 \tau^{2}}{4}\left(1-v^{2}\right)^{2} \\
+4\left(\frac{H^{2}+\tau^{2}}{\kappa-4 \tau^{2}}\right)\left(H^{2}-K_{e}\right)-\frac{q}{\kappa-4 \tau^{2}}
\end{gathered}
$$

and finally, re-ordering in terms of $H^{2}-K_{e}$, we obtain the first expression. 
Next, by differentiating (2.5) with respect to $\bar{z}$ and using (2.7), (2.4) and (2.3), one gets

$$
v_{z \bar{z}}=-\left(\kappa-4 \tau^{2}\right) v|A|^{2}-\frac{2}{\lambda}|p|^{2} v-\frac{H^{2}+\tau^{2}}{2} \lambda v .
$$

Then, from (2.6),

$$
v_{z \bar{z}}=-\frac{\lambda v}{4}\left(\left(\kappa-4 \tau^{2}\right)\left(1-v^{2}\right)+\frac{8|p|^{2}}{\lambda^{2}}+2\left(H^{2}+\tau^{2}\right)\right),
$$

thus

$$
\Delta v=\frac{4}{\lambda} v_{z \bar{z}}=-\left(\left(\kappa-4 \tau^{2}\right)\left(1-v^{2}\right)+2\left(H^{2}-K_{e}\right)+2\left(H^{2}+\tau^{2}\right)\right) \nu .
$$

Finally,

$$
\Delta \ln q=\Delta \ln \frac{4|Q|^{2}}{\lambda^{2}}=-2 \Delta \ln \lambda=4 K,
$$

where we have used that $Q d z^{2}$ is holomorphic and the expression of the Gaussian curvature in terms of a conformal parameter.

Remark 2.1. Note that (2.9) is nothing but the Jacobi equation for the Jacobi field $v$.

Next, we recall a definition in these homogeneous spaces.

Definition 2.1. We say that $\Sigma \subset \mathbb{E}(\kappa, \tau)$ is a vertical cylinder over $\alpha$ if $\Sigma=\pi^{-1}(\alpha)$, where $\alpha$ is a curve on $\mathbb{M}^{2}(\kappa)$.

It is not hard to verify that if $\alpha$ is a complete curve of geodesic curvature $2 H$ on $\mathbb{M}^{2}(\kappa)$, then $\Sigma=\pi^{-1}(\alpha)$ is complete and has constant mean curvature $H$. Moreover, these cylinders are characterized by $v \equiv 0$.

We now state two results about the classification of $H$-surfaces. They will be used in Sections 3 and 4, but we prove them in Section 5 and Section 6 for the sake of clarity. The first one concerns $H$-surfaces for which the angle function is constant. However, we need to introduce a family of surfaces that appear in the classification.

Definition 2.2. Denote by $S_{\kappa, \tau}$ a family of complete $H$-surfaces in $\mathbb{E}(\kappa, \tau), \kappa<0$, satisfying for any $\Sigma \in S_{\kappa, \tau}$ :

- $4 H^{2}+\kappa<0$.

- $q$ vanishes identically on $\Sigma \in S_{\kappa, \tau}$, i.e., $\Sigma$ is invariant by a one parameter family of isometries.

- $0<v^{2}<1$ is constant along $\Sigma$.

- $K_{e}=-\tau^{2}$ and $K=\left(\kappa-4 \tau^{2}\right) \nu^{2}<0$ are constants along $\Sigma$. 
An anonymous referee indicated to us the preprint "Hypersurfaces with a parallel higher fundamental form" by S. Verpoort who observed that we mistakenly omitted the surfaces $S_{\kappa, \tau}$ in a first draft of this paper.

Theorem 2.2. Let $\Sigma \subset \mathbb{E}(\kappa, \tau)$ be a complete $H$-surface with constant angle function. Then $\Sigma$ is either a vertical cylinder over a complete curve of curvature $2 H$ on $\mathbb{M}^{2}(\kappa)$, a slice in $\mathbb{H}^{2} \times \mathbb{R}$ or $\mathbb{S}^{2} \times \mathbb{R}$, or $\Sigma \in S_{\kappa, \tau}$ with $\kappa<0$.

Remark 2.2. Theorem 2.2 improves Lemma 2.3 in [ER] for surfaces in $\mathbb{H}^{2} \times \mathbb{R}$.

Of special interest for us are those $H$-surfaces for which the Abresch-Rosenberg differential is constant.

Theorem 2.3. Let $\Sigma \subset \mathbb{E}(\kappa, \tau)$ be a complete $H$-surface with $q$ constant.

- If $q=0$, then $\Sigma$ is invariant by a one-parameter group of isometries of $\mathbb{E}(\kappa, \tau)$, and if $H=0=\tau$, then $\Sigma$ is a slice in $\mathbb{H}^{2} \times \mathbb{R}$ or $\mathbb{S}^{2} \times \mathbb{R}$.

Moreover, the Gauss curvature of these examples is as follows.

- If $4 H^{2}+\kappa>0$, then $K=0$, and they are rotationally invariant spheres.

- If $4 H^{2}+\kappa=0$ and $v \equiv 0$, then $K \equiv 0$ and $\Sigma$ is either a vertical plane in $\mathrm{Nil}_{3}$, or a vertical cylinder over a horocycle in $\mathbb{H}^{2} \times \mathbb{R}$ or $\overline{\operatorname{PSL}(2, \mathbb{C})}$.

- There exists a point with negative Gauss curvature in the remaining cases.

- If $q \neq 0$ on $\Sigma$, then $\Sigma$ is a vertical cylinder over a complete curve of curvature $2 H$ on $\mathbb{M}^{2}(\kappa)$.

\section{Complete $H$-surfaces $\Sigma$ with $K \geq 0$}

Here we prove

Theorem 3.1. Let $\Sigma \subset \mathbb{E}(\kappa, \tau)$ be a complete $H$-surface with $K \geq 0$. Then, $\Sigma$ is either a rotational sphere (in particular, $4 H^{2}+\kappa>0$ ), or a complete vertical cylinder over a complete curve of geodesic curvature $2 \mathrm{H}$ on $\mathbb{M}^{2}(\kappa)$.

Proof. The proof goes as follows: First, we prove that $\Sigma$ is a topological sphere or a complete non-compact parabolic surface. We show that when the surface is a topological sphere then it is a rotational sphere. If $\Sigma$ is a complete non-compact parabolic surface, we prove that it is a vertical cylinder by means of Theorem 2.3.

Since $K \geq 0$ and $\Sigma$ is complete, Lemma 5 in [KO] implies that $\Sigma$ is either a sphere or non-compact and parabolic. 
If $\Sigma$ is a sphere, then it is a rotational example (see [AR2] or [AR]). Thus, we can assume that $\Sigma$ is non-compact and parabolic.

We can assume that $q$ does not vanish identically in $\Sigma$. If $q$ does vanish, then $\Sigma$ is either a vertical cylinder over a straight line in $\mathrm{Nil}_{3}$ or a vertical cylinder over a horocycle in $\mathbb{H}^{2} \times \mathbb{R}$ or $\widehat{\operatorname{PLS}(2, \mathbb{C})}$. Note that we have used here that $K \geq 0$ and Theorem 2.3.

On the one hand, from the Gauss equation (2.2)

$$
0 \leq K=K_{e}+\tau^{2}+\left(\kappa-4 \tau^{2}\right) \nu^{2} \leq K_{e}+\tau^{2}+\left|\kappa-4 \tau^{2}\right|,
$$

hence

$$
H^{2}-K_{e} \leq H^{2}+\tau^{2}+\left|\kappa-4 \tau^{2}\right| \text {. }
$$

On the other hand, using the very definition of $Q d z^{2},(3.1)$ and the inequality $\left|\xi_{1}+\xi_{2}\right|^{2} \leq 2\left(\left|\xi_{1}\right|^{2}+|\xi|^{2}\right)$ for $\xi_{1}, \xi_{2} \in \mathbb{C}$, we obtain

$$
\begin{aligned}
\frac{q}{2} & =\frac{2|Q|^{2}}{\lambda^{2}} \leq 4\left(H^{2}+\tau^{2}\right) \frac{4|p|^{2}}{\lambda^{2}}+\left(\kappa-4 \tau^{2}\right)^{2} \frac{4|A|^{4}}{\lambda^{2}} \\
& =4\left(H^{2}+\tau^{2}\right)\left(H^{2}-K_{e}\right)+\frac{\left(\kappa-4 \tau^{2}\right)^{2}}{4}\left(1-v^{2}\right)^{2} \\
& \leq 4\left(H^{2}+\tau^{2}\right)\left(H^{2}-K_{e}\right)+\frac{\left(\kappa-4 \tau^{2}\right)^{2}}{4} \\
& \leq 4\left(H^{2}+\tau^{2}\right)\left(H^{2}+\tau^{2}+\left|\kappa-4 \tau^{2}\right|\right)+\frac{\left(\kappa-4 \tau^{2}\right)^{2}}{4} .
\end{aligned}
$$

So, from (2.10), $\Delta \ln q=4 K \geq 0$ and $\ln q$ is a bounded subharmonic function on a non-compact parabolic surface $\Sigma$ and since the value $-\infty$ is allowed at isolated points (see $[\mathrm{AS}]$ ), $q$ is a positive constant (recall that we are assuming that $q$ does not vanish identically). Therefore, Theorem 2.3 gives the result.

\section{Complete $H$-surfaces $\Sigma$ with $K \leq 0$}

Theorem 4.1. Let $\Sigma \subset \mathbb{E}(\kappa, \tau)$ be a complete $H$-surface with $K \leq 0$ and $H^{2}+$ $\tau^{2}-\left|\kappa-4 \tau^{2}\right|>0$. Then, $\Sigma$ is a complete vertical cylinder over a complete curve of geodesic curvature $2 H$ on $\mathbb{M}^{2}(\kappa)$.

Proof. We divide the proof into two cases, $\kappa-4 \tau^{2}<0$ and $\kappa-4 \tau^{2}>0$.

Case $\kappa-4 \tau^{2}<0$ : On the one hand, since $K \leq 0$, we have

$$
H^{2}-K_{e} \geq H^{2}+\tau^{2}+\left(\kappa-4 \tau^{2}\right) \nu^{2} \geq H^{2}+\kappa-3 \tau^{2},
$$


from the Gauss equation (2.2). Therefore, from (2.8) and $\kappa-4 \tau^{2}<0$, we obtain:

$$
\begin{aligned}
q \geq 4 & \left(H^{2}+\tau^{2}\right)\left(H^{2}-K_{e}\right)+\left(\kappa-4 \tau^{2}\right)\left(1-v^{2}\right) \\
& \cdot\left(H^{2}+\tau^{2}+H^{2}-K_{e}+\frac{\kappa-4 \tau^{2}}{4}\left(1-v^{2}\right)\right) \\
= & \left(H^{2}-K_{e}\right)\left(4 H^{2}+4 \tau^{2}+\left(\kappa-4 \tau^{2}\right)\left(1-v^{2}\right)\right) \\
& +\left(H^{2}+\tau^{2}\right)\left(\kappa-4 \tau^{2}\right)\left(1-v^{2}\right)+\frac{\left(\kappa-4 \tau^{2}\right)^{2}}{4}\left(1-v^{2}\right)^{2} \\
\geq( & \left.H^{2}+\tau^{2}+\left(\kappa-4 \tau^{2}\right) v^{2}\right)\left(4 H^{2}+4 \tau^{2}+\left(\kappa-4 \tau^{2}\right)\left(1-v^{2}\right)\right) \\
& +\left(H^{2}+\tau^{2}\right)\left(\kappa-4 \tau^{2}\right)\left(1-v^{2}\right)+\frac{\left(\kappa-4 \tau^{2}\right)^{2}}{4}\left(1-v^{2}\right)^{2}
\end{aligned}
$$

note that the last inequality holds since $4 H^{2}+4 \tau^{2}+\left(\kappa-4 \tau^{2}\right)\left(1-v^{2}\right) \geq 4 H^{2}+\kappa>0$. $4 H^{2}+\kappa>0$ follows from

$$
0<4\left(H^{2}+\tau^{2}\right)-\left|\kappa-4 \tau^{2}\right|=4 H^{2}+\kappa .
$$

Set $a:=H^{2}+\tau^{2}$ and $b:=\kappa-4 \tau^{2}$. Define the real smooth function $f:[-1,1] \rightarrow$ $\mathbb{R}$ as

$$
f(x)=\left(a+b x^{2}\right)\left(4 a+b\left(1-x^{2}\right)\right)+a b\left(1-x^{2}\right)+\frac{b^{2}}{4}\left(1-x^{2}\right)^{2} .
$$

Note that $q \geq f(v)$ on $\Sigma, f(v)$ is just the last part in the above inequality involving $q$. It is easy to verify that the only critical point of $f$ in $(-1,1)$ is $x=0$. Moreover,

$$
f(0)=(4 a+b)^{2} / 4>0 \text { and } \quad f( \pm 1)=4 a(a+b)>0 .
$$

Actually, $f: \mathbb{R} \rightarrow \mathbb{R}$ has two others critical points, $x= \pm \sqrt{\frac{4 a+b}{3|b|}}$, but here we have used that

$$
\frac{4 a+b}{3|b|}>1
$$

since $0<4\left(H^{2}+\kappa-3 \tau^{2}\right)=\left(4 H^{2}+\kappa\right)-3\left|\kappa-4 \tau^{2}\right|=(4 a+b)-3|b|$.

So, set $c=\min \{f(0), f( \pm 1)\}>0$, then

$$
q \geq f(v) \geq c>0
$$

Now, from (2.10) and $q \geq c>0$ on $\Sigma$, it follows that $d s^{2}=\sqrt{q} I$ is a complete flat metric on $\Sigma$ and

$$
\Delta^{d s^{2}} \ln q=\frac{1}{\sqrt{q}} \Delta \ln q=\frac{4 K}{\sqrt{q}} \leq 0
$$


Since $q$ is bounded below by a positive constant and $\left(\Sigma, d s^{2}\right)$ is parabolic, then $\ln q$ is constant which implies that $q$ is a positive constant. Thus, the result follows from Theorem 2.3. The case $\kappa-4 \tau^{2}<0$ is proved.

Case $\kappa-4 \tau^{2}>0$ : Set $w_{1}:=2(H+i \tau) \frac{p}{\lambda}$ and $w_{2}:=\left(\kappa-4 \tau^{2}\right) \frac{A^{2}}{\lambda}$, i.e., $q=$ $4\left|w_{1}-w_{2}\right|^{2}$. Then

$$
\begin{aligned}
& \left|w_{1}\right|^{2}=\left(H^{2}+\tau^{2}\right)\left(H^{2}-K_{e}\right) \geq\left(H^{2}+\tau^{2}\right)^{2}, \\
& \left|w_{2}\right|^{2}=\frac{\left(\kappa-4 \tau^{2}\right)^{2}}{16}\left(1-v^{2}\right)^{2} \leq\left(\frac{\kappa-4 \tau^{2}}{4}\right)^{2},
\end{aligned}
$$

where we have used that $H^{2}-K_{e} \geq H^{2}+\tau^{2}+\left(\kappa-4 \tau^{2}\right) v^{2} \geq H^{2}+\tau^{2}$, since $K \leq 0$ and $\kappa-4 \tau^{2}>0$.

We recall a well-known inequality for complex numbers. Let $\xi_{1}, \xi_{2} \in \mathbb{C}$, then $\left|\xi_{1}+\xi_{2}\right|^{2} \geq|| \xi_{1}|-| \xi_{2}||^{2}$. Thus,

$$
\begin{aligned}
\frac{1}{4} q & \geq|| w_{1}|-| w_{2}||^{2} \geq\left|\left(H^{2}+\tau^{2}\right)-\frac{\left|\kappa-4 \tau^{2}\right|}{4}\right|^{2} \\
& =\frac{1}{16}\left|4\left(H^{2}+\tau^{2}\right)-\right| \kappa-4 \tau^{2}||^{2}>0 .
\end{aligned}
$$

So, as $q$ is bounded below by a positive constant, then, arguing as in the previous case, $q$ is a constant. Thus, the result follows from Theorem 2.3. The case $\kappa-4 \tau^{2}>0$ is proved.

Remark 4.1. Note that in the above theorem, in the case $\kappa-4 \tau^{2}>0$, we only need to assume that $4\left(H^{2}+\tau^{2}\right)-\left|\kappa-4 \tau^{2}\right|>0$.

\section{Complete $\boldsymbol{H}$-surfaces with constant angle function}

We classify here the complete $H$-surfaces in $\mathbb{E}(\kappa, \tau)$ with constant angle function. The purpose is to take advantage of this classification result in the next section.

Theorem 2.2. Let $\Sigma \subset \mathbb{E}(\kappa, \tau)$ be a complete $H$-surface with constant angle function. Then $\Sigma$ is either a vertical cylinder over a complete curve of curvature $2 H$ on $\mathbb{M}^{2}(\kappa)$, a slice in $\mathbb{H}^{2} \times \mathbb{R}$ or $\mathbb{S}^{2} \times \mathbb{R}$, or $\Sigma \in \mathcal{S}_{\kappa, \tau}$ with $\kappa<0$ (see Definition 2.2).

Proof. We can assume that $v \leq 0$. We will divide the proof into three cases:

- $v=0$ : In this case, $\Sigma$ must be a vertical cylinder over a complete curve of geodesic curvature $2 H$ on $\mathbb{M}^{2}(\kappa)$. 
- $v=-1$ : From (2.4), $\tau=0$ and $H=0$, then $\Sigma$ is a slice in $\mathbb{H}^{2} \times \mathbb{R}$ or $\mathbb{S}^{2} \times \mathbb{R}$.

- $-1<v<0$ : We prove here that $\Sigma \in S_{\kappa, \tau}$ with $\kappa<0$. From (2.5) we have

$$
(H-i \tau) A=-\frac{2 p}{\lambda} \bar{A},
$$

then

$$
H^{2}+\tau^{2}=\frac{4|p|^{2}}{\lambda^{2}}=H^{2}-K_{e}
$$

since $|A|^{2} \neq 0$ from (2.6), so $K_{e}=-\tau^{2}$ on $\Sigma$.

Thus, from (2.9), we have

$$
4 H^{2}+4 \tau^{2}+\left(\kappa-4 \tau^{2}\right)\left(1-v^{2}\right)=0 .
$$

Now, using the definition of $q,(5.1),(5.2)$ and $K_{e}=-\tau^{2}$, we have

$$
\begin{gathered}
q=\frac{4|Q|^{2}}{\lambda^{2}}=4\left(H^{2}+\tau^{2}\right) \frac{4|p|^{2}}{\lambda^{2}}+\left(\kappa-4 \tau^{2}\right)^{2} \frac{4|A|^{4}}{\lambda^{2}} \\
\quad-4 \frac{\kappa-4 \tau^{2}}{\lambda^{2}}\left(2(H+i \tau) p \bar{A}^{2}+2(H-i \tau) \bar{p} A^{2}\right) \\
=4\left(H^{2}+\tau^{2}\right)\left(H^{2}-K_{e}\right)+\left(\kappa-4 \tau^{2}\right)^{2} \frac{\left(1-v^{2}\right)^{2}}{4} \\
\quad+2\left(\kappa-4 \tau^{2}\right)\left(1-v^{2}\right)\left(H^{2}+\tau^{2}\right) \\
=\frac{1}{4}\left(4 H^{2}+\left(\kappa-4 \tau^{2}\right)\left(1-v^{2}\right)+4 \tau^{2}\right)^{2}=0,
\end{gathered}
$$

that is, $q$ vanishes identically on $\Sigma$. Moreover, from (5.2), we can see that $4 H^{2}+\kappa<0$, that is, $\kappa<0$. Therefore, $\Sigma \in S_{\kappa, \tau}, \kappa<0$.

\section{Complete $H$-surfaces with $q$ constant}

Here, we prove the classification result for complete $H$-surfaces in $\mathbb{E}(\kappa, \tau)$ employed in the proof of Theorem 3.1 and Theorem 4.1.

Theorem 2.3. Let $\Sigma \subset \mathbb{E}(\kappa, \tau)$ be a complete $H$-surface with $q$ constant.

- If $q=0$ on $\Sigma$, then $\Sigma$ is either a slice in $\mathbb{H}^{2} \times \mathbb{R}$ or $\mathbb{S}^{2} \times \mathbb{R}$ if $H=0=\tau$, or $\Sigma$ is invariant by a one-parameter group of isometries of $\mathbb{E}(\kappa, \tau)$.

Moreover, the Gauss curvature of these examples is as follows.

- If $4 H^{2}+\kappa>0$, then $K>0$ they are the rotationally invariant spheres. 
- If $4 H^{2}+\kappa=0$ and $v \equiv 0$, then $K \equiv 0$ and $\Sigma$ is either a vertical plane in $\mathrm{Nil}_{3}$, or a vertical cylinder over a horocycle in $\mathbb{H}^{2} \times \mathbb{R}$ or $\widehat{\operatorname{PSL}(2, \mathbb{C})}$.

- There exists a point with negative Gauss curvature in the remaining cases.

- If $q \neq 0$ on $\Sigma$, then $\Sigma$ is a vertical cylinder over a complete curve of curvature $2 H$ on $\mathbb{M}^{2}(\kappa)$.

The case $q=0$ has been treated extensively when the target manifold is a product space, but is has not been established explicitly when $\tau \neq 0$. So, we assemble the results in [AR], [AR2] for the reader's convenience.

Lemma 6.1. Let $\Sigma \subset \mathbb{E}(\kappa, \tau)$ be a complete $H$-surface whose Abresch-Rosenberg differential vanishes. Then $\Sigma$ is either a slice in $\mathbb{H}^{2} \times \mathbb{R}$ or $\mathbb{S}^{2} \times \mathbb{R}$ if $H=0=\tau$, or $\Sigma$ is invariant by a one-parameter group of isometries of $\mathbb{E}(\kappa, \tau)$.

Moreover, the Gauss curvature of these examples is as follows.

- If $4 H^{2}+\kappa>0$, then $K>0$ they are the rotationally invariant spheres.

- If $4 H^{2}+\kappa=0$ and $v \equiv 0$, then $K \equiv 0$ and $\Sigma$ is either a vertical plane in $\mathrm{Nil}_{3}$, or a vertical cylinder over a horocycle in $\mathbb{H}^{2} \times \mathbb{R}$ or $\widehat{\operatorname{PSL}(2, \mathbb{C})}$.

- There exists a point with negative Gauss curvature in the remaining cases.

Proof. The idea of the proof for product spaces that we use below can be found in $[\mathrm{dCF}]$ and $[\mathrm{FM}]$.

If $H=0=\tau$, from the definition of the Abresch-Rosenberg differential, we have

$$
0=-(\kappa-4 \tau) A^{2}
$$

that is, $v^{2}= \pm 1$ using (2.6). Thus, $\Sigma$ is a slice in $\mathbb{H}^{2} \times \mathbb{R}$ or $\mathbb{S}^{2} \times \mathbb{R}$.

If $H \neq 0$ or $\tau \neq 0$, we have

$$
2(H+i \tau) p=\left(\kappa-4 \tau^{2}\right) A^{2},
$$

from where we obtain, taking modulus,

$$
H^{2}-K_{e}=\frac{\left(\kappa-4 \tau^{2}\right)^{2}\left(1-v^{2}\right)^{2}}{16\left(H^{2}+\tau^{2}\right)} .
$$

Inserting (6.1) in (2.5),

$$
(H+i \tau) v_{z}=-\frac{1}{4}\left(4 H^{2}+\kappa-\left(\kappa-4 \tau^{2}\right) v^{2}\right) A,
$$

and taking modulus,

$$
\left|v_{z}\right|^{2}=g(v)^{2}|A|^{2}, \quad g(v)=\frac{4 H^{2}+\kappa-\left(\kappa-4 \tau^{2}\right) v^{2}}{4 \sqrt{H^{2}+\tau^{2}}} .
$$


Assume that $v$ is not constant. Let $p \in \Sigma$ be a point where $v_{z}(p) \neq 0$ and let $U$ be a neighborhood of that point $p$ where $v_{z} \neq 0$ (we can assume $v^{2} \neq 1$ at $p$ ). In particular, $g(v) \neq 0$ in $U$ from (6.3). Now, inserting (6.3) in (2.6), we obtain

$$
\lambda=\frac{4\left|v_{z}\right|^{2}}{\left(1-v^{2}\right) g(v)^{2}} .
$$

Thus, putting (6.2) and (6.4) in the Jacobi equation (2.9)

$$
v_{z \bar{z}}=-2 \frac{v\left|v_{z}\right|^{2}}{1-v^{2}}
$$

So, define the real function $s:=\operatorname{arctgh}(v)$ on $U$. Such a function is harmonic by means of (6.5), thus we can consider a new conformal parameter $w$ for the first fundamental form so that $s=\operatorname{Re}(w), w=s+i t$.

Since $v=\operatorname{tgh}(s)$ by the definition of $s$, we have that $v \equiv v(s)$, i.e., it only depends on one parameter. Thus, we have $\lambda \equiv \lambda(s)$ and $T \equiv T(s)$ from (6.4) and (6.3) respectively, and $p \equiv p(s)$ by the definition of the Abresch-Rosenberg differential. That is, all the fundamental data of $\Sigma$ depend only on $s$.

Now, let $\mathcal{U}$ be a simply connected domain on $\Sigma$ and $\mathcal{V} \subset \mathbb{R}^{2}$ a simply connected domain of a surface $S$ so that $\psi_{0}: \mathcal{V} \rightarrow U \subset \mathbb{E}(\kappa, \tau)$. We parametrize $\mathcal{V}$ by the parameters $(s, t)$ obtained above. Then, the fundamental data (see [FM], Theorem 2.3) $\left\{\lambda_{0}, p_{0}, T_{0}, \nu_{0}\right\}$ of $\psi_{0}$ are given by

$$
\left\{\begin{array}{l}
\lambda_{0}(s, t)=\lambda(s), \\
p_{0}(s, t)=p(s), \\
T_{0}(s, t)=a(s) \partial_{s}, \\
v_{0}(s, t)=v(s),
\end{array}\right.
$$

where $a(s)$ is a smooth function.

Let $\bar{t} \in \mathbb{R}$ and let $\mathbf{i}_{\bar{t}}: \mathbb{R}^{2} \rightarrow \mathbb{R}^{2}$ be the diffeomorphism given by

$$
\mathbf{i}_{\bar{t}}(s, t):=(s, t+\bar{t}),
$$

and define $\psi_{\bar{t}}:=\psi_{0} \circ \mathbf{i}_{\bar{t}}$. Then, the fundamental data $\left\{\lambda_{\bar{t}}, p_{\bar{t}}, T_{\bar{t}}, v_{\bar{t}}\right\}$ of $\psi_{\bar{t}}$ are given by

$$
\left\{\begin{array}{l}
\lambda_{\bar{t}}(s, t)=\lambda(s), \\
p_{\bar{t}}(s, t)=p(s), \\
T_{\bar{t}}(s, t)=a(s) \partial_{s}, \\
v_{\bar{t}}(s, t)=v(s),
\end{array}\right.
$$

that is, both fundamental data match at any point $(s, t) \in \mathcal{V}$. Therefore, using [D], Theorem 4.3, there exists an ambient isometry $\mathcal{I}_{\bar{t}}: \mathbb{E}(\kappa, \tau) \rightarrow \mathbb{E}(\kappa, \tau)$ so that

$$
\mathcal{I}_{\bar{t}} \circ \psi_{0}=\psi_{0} \circ \mathbf{i}_{\bar{t}} \quad \text { for all } \bar{t} \in \mathbb{R},
$$


thus the surface is invariant by a one parameter group of isometries.

Let us prove the claim about the Gauss curvature. Using the Gauss equation (2.2) in (6.2), one gets

$$
H^{2}+\tau^{2}+\left(\kappa-4 \tau^{2}\right) v^{2}-K=\frac{\left(\kappa-4 \tau^{2}\right)^{2}\left(1-v^{2}\right)^{2}}{16\left(H^{2}+\tau^{2}\right)} .
$$

Set $a:=4\left(H^{2}+\tau^{2}\right)$ and $b:=\kappa-4 \tau^{2}$, then one can check easily that the above equality can be expressed as

$$
4 a K=a^{2}-b^{2}+(2 a+b)^{2}-\left(2 a+b\left(1-v^{2}\right)\right)^{2} .
$$

So, if $4 H^{2}+\kappa>0$ then $a>|b|$ and $K>0$, that is, $\Sigma$ is a topological sphere since it is complete. If $4 H^{2}+\kappa=0, a=-b$ and the equation reads as

$$
4 a K=a^{2}\left(1-\left(1+v^{2}\right)^{2}\right),
$$

that is, $\Sigma$ has a point with negative Gauss curvature unless $v \equiv 0$.

If $4 H^{2}+\kappa<0$, one can check that $a^{2}-b^{2}=(a-b)(a+b)<0$ since $a+b>0$ and $a-b<0$. So, if $\inf _{\Sigma}\left\{v^{2}\right\}=0$ then, from (6.6), $\Sigma$ has a point with negative curvature. Therefore, to finish this lemma, we shall prove the following

Claim. There are no complete constant mean curvature surfaces in $\mathbb{E}(\kappa, \tau)$ with $4 H^{2}+\kappa<0, q \equiv 0, K \geq 0$, and $\inf \left\{v^{2}\right\}=c>0$.

Proof of the Claim. Assume such a surface $\Sigma$ exists. Since we are assuming that $K \geq 0$ and $\Sigma$ is complete, then $\Sigma$ is parabolic and noncompact. If $\Sigma$ were compact we would have a contradiction with the fact that $\inf _{\Sigma}\left\{v^{2}\right\}=c>0$ and $4 H^{2}+\kappa<0$.

Since $q$ vanishes identically on $\Sigma, \operatorname{arctanh}(v)$ is a bounded harmonic function on $\Sigma$ and so $v$ is constant. So, the projection $\pi: \Sigma \rightarrow \mathbb{M}^{2}(\kappa)$ is a global diffeomorphism and a quasi-isometry. This is impossible since $\Sigma$ is parabolic and $\mathbb{M}^{2}(\kappa), \kappa<0$, is hyperbolic. Therefore, the Claim is proved and so the lemma is proved.

Proof of Theorem 2.3. We focus on the case $q \neq 0$ because Lemma 6.1 gives the classification when $q=0$.

Suppose $v$ is not constant in $\Sigma$. Since $q=c^{2}>0$, we can consider a conformal parameter $z$ so that $\langle\cdot, \cdot\rangle=|d z|^{2}$ and $Q d z^{2}=c d z^{2}$ on $\Sigma$. Thus,

$$
Q=c=2(H+i \tau) p-\left(\kappa-4 \tau^{2}\right) A^{2} .
$$

First, note that we can assume that $H \neq 0$ or $\tau \neq 0$, otherwise $v$ would be constant. So, from (2.5), we have

$$
(H+i \tau) v_{z}=-\left(H^{2}+\tau^{2}+\frac{\kappa-4 \tau^{2}}{4}\left(1-v^{2}\right)\right) A-c \bar{A},
$$


where we have used $2(H+i \tau) p=c+\left(\kappa-4 \tau^{2}\right) A^{2}$. That is,

$$
16\left(H^{2}+\tau^{2}\right)\|\nabla v\|^{2}=(g(v)+4 c)^{2}\left(1-v^{2}\right),
$$

where

$$
g(v):=4 H^{2}+\kappa-\left(\kappa-4 \tau^{2}\right) v^{2} .
$$

From (2.10), $\Sigma$ is flat and $H^{2}-K_{e}=H^{2}+\tau^{2}+\left(\kappa-4 \tau^{2}\right) v^{2}$ by (2.2), joining this last equation to (2.8) we obtain using the definition of $g(v)$ given in (6.8)

$$
\|\nabla v\|^{2}=\frac{g(v)^{2}}{4\left(\kappa-4 \tau^{2}\right)}+v^{2} g(v)-\frac{c^{2}}{\kappa-4 \tau^{2}} .
$$

Putting together (6.7) and (6.9) we obtain a polynomial expression in $v^{2}$ with coefficients depending on $a:=4\left(H^{2}+\tau^{2}\right), b:=\kappa-4 \tau^{2}$ and $c$ :

$$
P\left(v^{2}\right):=C(a, b, c) v^{6}+\text { lower terms }=0,
$$

but one can easily check that the coefficient of $v^{6}$ is $C(a, b, c)=-a^{-1} b^{2} \neq 0$, a contradiction. Thus $v$ is constant, and so, by means of Theorem $2.2, \Sigma$ is a vertical cylinder over a complete curve of curvature $2 \mathrm{H}$.

\section{Appendix}

Let $\Sigma$ be a connected Riemannian surface. We establish in this Appendix a result which we think is of independent interest, concerning differential operators of the form $\Delta+g$, acting on $C^{2}(\Sigma)$-functions, where $\Delta$ is the Laplacian with respect to the Riemannian metric on $\Sigma$ and $g \in C^{0}(\Sigma)$.

Lemma 7.1. Let $g \in C^{0}(\Sigma), v \in C^{2}(\Sigma)$ such that $\|\nabla v\|^{2} \leq h v^{2}$ on $\Sigma, h$ is a non-negative continuous function on $\Sigma$, and $\Delta v+g v=0$ in $\Sigma$. Then either $v$ never vanishes or $v$ vanishes identically on $\Sigma$.

Proof. Set $\Omega=\{p \in \Sigma: v(p)=0\}$. We will show that either $\Omega=\emptyset$ or $\Omega=\Sigma$.

So, let us assume that $\Omega \neq \emptyset$. If we prove that $\Omega$ is an open set then, since $\Omega$ is closed and $\Sigma$ is connected, $\Omega=\Sigma$. Let $p \in \Omega$ and $\mathcal{B}(R) \subset \Sigma$ be the geodesic ball centered at $p$ of radius $R$. Such a geodesic ball is relatively compact in $\Sigma$.

Set $\phi=v^{2} / 2 \geq 0$. Then

$$
\Delta \phi=v \Delta v+\|\nabla v\|^{2}=-g v^{2}+\|\nabla v\|^{2} \leq-2(g-h) \phi,
$$

that is,

$$
-\Delta \phi-2(g-h) \phi \geq 0
$$


Define $\beta:=\min \left\{\inf _{\Omega}\{2(g-h)\}, 0\right\} \leq 0$. Then, $\psi=-\phi$ satisfies

$$
\Delta \psi+\beta \psi=-\Delta \phi-\beta \phi \geq-\Delta \phi-2(g-h) \phi \geq 0,
$$

where we have used (7.1).

Since we are assuming that $v$ has a zero at an interior point of $\mathcal{B}(R), \beta \leq 0$ and $\psi$ has a non-negative maximum at $p$, the Maximum Principle [GT], Theorem 3.5, implies that $\psi$ is constant and so $v$ is constant as well, i.e, $v \equiv 0$ in $\mathcal{B}(R)$. Then $\mathscr{B}(R) \subset \Omega$, and $\Omega$ is an open set. Thus $\Omega=\Sigma$.

\section{References}

[AS] L. Ahlfors and L. Sario, Riemann surfaces. Princeton Math. Ser. 26, Princeton University Press, Princeton, N.J., 1960. Zbl 0196.33801 MR 0114911665

[AR] U. Abresch and H. Rosenberg, A Hopf differential for constant mean curvature surfaces in $\mathbb{S}^{2} \times \mathbb{R}$ and $\mathbb{H}^{2} \times \mathbb{R}$. Acta Math. 193 (2004), 141-174. Zbl 1078.53053 MR 2134864 $659,661,665,669$

[AR2] U. Abresch, H. Rosenberg, Generalized Hopf differentials. Mat. Contemp. 28 (2005), 1-28. Zbl 1118.53036 MR 2195187 659, 661, 665, 669

[Ch] S. Y. Cheng, Eigenfunctions and nodal sets. Comment. Math. Helv. 51 (1976), 43-55. Zbl 0334.35022 MR 0397805

[CoR] P. Collin and H. Rosenberg, Construction of harmonic diffeomorphisms and minimal graphs. Ann. of Math. (2) 172 (2010), 1879-1906. Zbl 1209.53010 MR 2726102659

[D] B. Daniel, Isometric immersions into 3-dimensional homogenouos manifolds. Comment. Math. Helv. 82 (2007), 87-131. Zbl 1123.53029 MR 2296059670

[DH] B. Daniel and L. Hauswirth, Half-space theorem, embedded minimal annuli and minimal graphs in the Heisenberg group. Proc. Lond. Math. Soc. (3) 98 (2009), 445-470. Zbl 1163.53036 MR 2481955659

[dCF] M. do Carmo and I. Fernández, A Hopf theorem for open surfaces in product spaces. Forum Math. 21 (2009), 951-963. Zbl 1188.53072 MR 2574142669

[ER] J. M. Espinar and H. Rosenberg, Complete constant mean curvature surfaces and Bernstein type theorems in $\mathbb{M}^{2} \times \mathbb{R}$. J. Differential Geom. 82 (2009), 611-628. Zbl 1180.53062 MR 2534989 659, 664

[FM] I. Fernández and P. Mira, A characterization of constant mean curvature surfaces in homogeneous three-manifolds. Differential Geom. Appl. 25 (2007), 281-289. Zbl 1125.53007 MR 2330457 659, 661, 669, 670

[FM2] I. Fernández and P. Mira, Holomorphic quadratic differentials and the Bernstein problem in Heisenberg space. Trans. Amer. Math. Soc. 361 (2009), 5737-5752. Zbl 05626126 MR 2529912659

[GT] D. Gilbarg and N. S. Trudinger, Elliptic partial differential equations of second order. 2nd edition, Springer-Verlag, New York, 1983. Zbl 0562.35001 MR 0737190673 
[H] D. Hoffman, Surfaces of constant mean curvature in manifolds of constant curvature. $J$. Differential Geom. 8 (1973), 161-176. Zbl 0284.53040 MR 0390973659

[KO] T. Klotz, R. Osserman, Complete surfaces in $E^{3}$ with constant mean curvature. Comment. Math. Helv. 41 (1966-67), 313-318. Zbl 0163.16002 MR 0211332 659, 664

[T] R. Tribuzy, Hopf's method and deformations of surfaces preserving mean curvature. An. Acad. Brasil. Ciênc. 50 (1978), 447-450. Zbl 0404.53047 MR 0525768659

Received March 14, 2009

José M. Espinar, Departamento de Geometría y Topología, Universidad de Granada, Avda Fuentenueva s/n, 18071 Granada, Spain

E-mail: jespinar@ugr.es

Harold Rosenberg, Instituto de Matematica Pura y Aplicada, 110 Estrada Dona Castorina, Rio de Janeiro 22460-320, Brazil

E-mail: rosen@impa.br 